\section{Training in Forensic Microscopy}

William Chapin, Johnson County Crime Lab, Mission, KS

The world of forensic microscopy is complex and challenging. There is a never-ending variety of requested examinations and consequently, the analyst is often asked to work in an area of little familiarity. Adding to the challenge is the inherent problem of having no exact information as to the condition of the sample prior to the crime. The forensic microscopist is expected to have mastered proficiency in polarized light microscopy in order to identify fibers, minerals, insect parts, woods, metals, biological fluids, pollution particles, microchemical techniques, paints, pigments, pollens, drugs, explosives, diatoms, safe insulation, etc. and to compare samples of all the these plus hairs, glass, etc., etc.

He/she must analyze a sample from an unknown environment, with a questionable history, and testify in a court of law as to conclusions with absolute certainty. With time and commitment, one could conceivably become comfortable doing this type of work. That is, until other job junctions are factored into the equation - proficiency using numerous types of instrumentation, instrumental repair, crime scene analysis, courtroom testimony, administrative tasks, professional organization activities, research and subsequent paper witing, teaching, laboratory analyses not involving microscopy, and the list goes on ad nauseam.

In most forensic laboratories there is an understandable frustration concerning the future of light microscopy. This frustration coupled with the natural desire for "bigger and newer" instrumentation has caused traditional microscopy to fall into a state of near disuse. Unfortunately, though these new instruments are "bigger and newer" they are also more costly and often yield no more and sometimes less valuable information for the higher price tag.

It is a bleak picture and one that has shown little sign of improvement until the past few years. The question posed to the few existing dyed-inthe-wool forensic microscopists is: "How can we improve the current state of affairs?" To gain insight in answering that question, a particular laboratory system is used as an example. In 1986, the Johnson County Crime Laboratory, Mission, Kansas, hired a new drug chemist so that the existing drug chemist could begin doing a more thorough job of analyzing "trace evidence"

"Trace evidence" is a loose term used to describe the analysis of paint, glass, fibers, explosive residues, soils, hair, and an undefined area consisting of the analysis of any physical articles that may be helpful in documenting a crime, substantiating the events of a crime or linking a suspect to a crime. When the change occurred, no major expenditures for new equipment were possible. The laboratory was fortunately blessed with a couple of decent microscopes and consent was granted to commit limited funds to purchase additional microscopy equipment.

There was also a laboratory history of providing good training. The obvious place to begin microscopy training (the only (?) place to receive microscopy training) was the McCrone Research Institute. Quite frankly, the price tag was a problem for a government agency. There was an attitude of "anyone can look through a microscope and see what's there, so why is the cost so high?" Still, when we consider costs of more than $\$ 1,000 /$ semester hour for University courses maybe McRl isn't out of line.

Obviously, an educational process had to occur within the administration. The issue to address centered around the perceived value for the dollars expended. Specific areas needing to be addressed were: the complexity and variety of theories to be understood, the knowledge of a diverse array of equipment needed to address the varied requested analyses, and the accuracy and extent of information that can be generated utilizing microscopical techniques. Once the above issues are effectively addressed, it is appreciated that training costs can replace expensive new instrumentation costs. When appropriate training is provided, the resulting microscopical analysis will generally be of a higher quality and more useful to the judicial system than analyses obtained with many types of instrumentation.

The situation was addressed in Johnson County by first hosting an on-site McCrone Basic Polarized Light Microscopy Course. It proved relatively easy to put together. Finding a cost-effective hotel with the proper facilities was the first step. It was then a simple matter to work with McRI to arrange timing and financial details. Lastly, letters were witten to generate a class. It was all put together in such a way that the Johnson County Criminalistics Laboratory handled no money and was able to put two of their own people in the class. That course set the stage for additional training.

Once the analyst begins to understand the potential of the microscope and is able to demonstrate it to others, both in and out of the laboratory, the logjam is broken. The analyst becomes more aware of casework applications and makes sure the administration is aware of the type of information being generated.

Secondly, the administration was requested to authorize training dealing in issues arising from casework. Courses such as Microscopy of Inorganic Explosives were attended and immediately put to use. Spring symposia of the $\frac{\overrightarrow{0}}{2}$ Minnesota Society of Optical Microscopy were attended. Over the next three

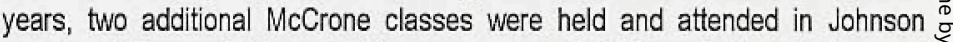
county: Advanced Forensic Microscopy and Soil Mineral Identification and Comparison. Other workshops were also attended through MAFS and the FBI $\frac{3}{0}$ Training Facility in Quantico, Virginia.

This training has been invaluable, but deficiencies still exist. After seven $\stackrel{0}{5}$ years, several topics have not yet been addressed (pollens, entomology, diatoms, pollution particles, seeds and fruiting bodies, wood identification, pigments and certain of the microscopical techniques). In some instances the training has not been optimally implemented. The problem of finding time away from casework for experimentation and technique development is acute. When a particular technique is to be employed in casework, there is often a short time of trial and error in an attempt to develop the necessary skills needed to effectively utilize the technique. This occurs within the context of the case and can easily be influenced by the expected results with that particular sample. This is certainly not a optimum environment in which to be developing good scientific techniques. In forensic analysis, there is often too little sample, often analysis can be a one-particle affair. The stakes are high and the focus tends to be shortsighted for the problem at hand.

There is another facet to the complexity of the forensic microscopist's task and necessary training. The required analyses cannot always be performed with one or even two microscopical techniques. There are a multitude of microscope types and even more accessory techniques for particle analysis: - consider hotstage microscopy, phase contrast, Hoffman modulation, differential interference contrast, darkfield, dispersion staining, fluorescence, and confocal microscopy. Other sophisticated "instrumental" microscopy techniques of, at least, potential forensic use include scanning electron microscopy (SEM) [often this is in conjunction with energy dispersive $\mathrm{X}$-ray analysis (EDX)], X-ray microscopy, FTIR Microscopy, analytical transmission electron microscopy (ATEM), electron microprobe analyzer (EMA), molecular optics laser examiner (MOLE), and the list goes on

There is little or no expection of becoming proficient in most of the above fields; however, one must have enough knowledge to recognize their potential usefulness in particular situations. Consequently, training at least in the theory of operation for each technique is desirable.

With all of the microscopical techniques there are associated techniques that also require training. Particle handling and sample preparation is sa skill that must be taught and practiced. Also, one must be schooled and have a working knowledge of photomicrography and image processing (video enhancement and documentation) equipment and techniques.

What can microscopists in general do to ease the training problems in forensic microscopy? First, more individuals can assist in providing local training on a cost effective basis. There are certainly more individuals qualified to teach than are stepping forward to offer workshops. This paper is an example of stepping forward. The author has had only a few years experience and certainly feels inadequate but, at the same time, he is impressed enough by the state of affairs to risk presenting this material in the hope that additional professionals in the field of microscopy will respond.

Second, we can follow the Document Examiners' approach. They may do less research but they do more sharing of general casework. . There are limited textbook answers to the forensic microscopists' problems of dealing with varied sample matrices. Even the experiences of a novice's casework may prove 
beneficial to the time-worn professional in unraveling a problem-sample analysis.

Third, anyone can do the legwork necessary to set up training. Bring in an expert, find a class, and reap the benefits of the training and the admiration of peers for providing the training. Another avenue is to work through one of the professional organizations. For example, MAFS has an extensive Training and Education Program with a continuing need for both coordinates and instructors.

Fourth, be a salesman of the discipline. The biggest obstacle is ignorance concerning what microscopists can do and what is required to accomplish the task. Talk about successes, show off impressive slides of work performed, and openly discuss the deficiencies in training. Work hard to do what the instrumentalists cannot do, and don't be afraid to "toot your horn".

A fifth practice is that of organizing a system of providing others with unknown samples. This is not meant to be formal, but it is a practice that can be extremely valuable to everyone in the network. Try sending the samples with a sealed envelop containing sample information so the recipient can work at his/her own pace and use the sample information at his/her discretion.

Lastly, the field of microscopy needs to be considered as a whole. As an example, industrial microscopists have much to offer forensic microscopists and vice versa. All of us in the different areas of microscopy should be communicating and assisting one another. This happens on a limited basis in meeting like INTER/MICRO and in selected geographical areas like Minneapolis/St. Paul, but I am convinced it does not occur often enough in many local areas. All if these suggestions have worked in Johnson County.

Other aspects that have not yet been addressed are patience and perspective. Look against what the goals are! They simply cannot be met overnight. It will take years of training and practice to do the job expected of a forensic microscopist. Patiently collect training courses, and attend any course that is available (there are no useless courses). While collecting the training, keep in perspective the skills that have been developed. Use those skills to their fullest, but be very careful not to push them beyond their limits. A great deal of harm can be brought to the field of microscopy if overstated conclusions are made. More specific to forensic microscopy, overstating conclusions could mean an embarrassing court testimony and possibly the end of a career. When in doubt, reevaluate, re-test, and communicate with other microscopists for their opinion.

Collect and analyze everything available (mount pollens, evaluate dog's hair, describe the botanical characteristics of kitchen spices, characterize the minerals in garden soil, identify everything found in various dust balls, analyze the fibers from your tie every day for 2 weeks, etc.) The forensic microscopist needs to be almost compulsively inquisitive about "particles". Collect and prepare standard slides of fibers, minerals, pollens, etc. and procure those available commercially

Forensic microscopy is making a slow comeback as people again recognize its power, but it is in a perilous state as it seems always on the verge of sinking into oblivion. It is much too valuable to lose. Microscopists must continue to educate one another and the administrations for which they work. Training is broad in scope and by no means as simple as many would like to believe. Once the educational process of the administration has been addressed, the training received by the analyst must be provided at the most reasonable cost possible. If one can ever feel fully trained, it will take years of dedication to the field and a considerable amount of "coursework". However, even with limited training, useful information can be generated that may not be available through the use of other instrumental techniques.

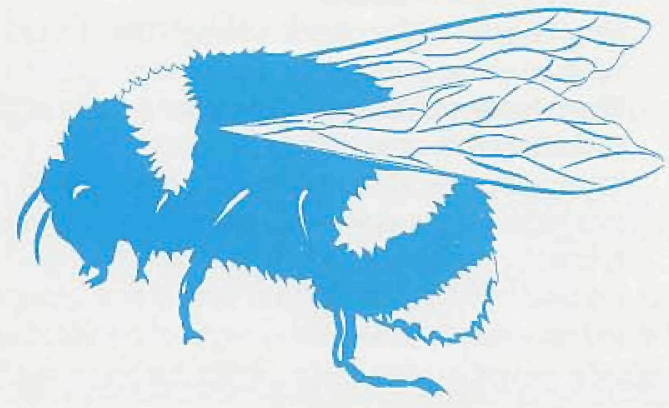

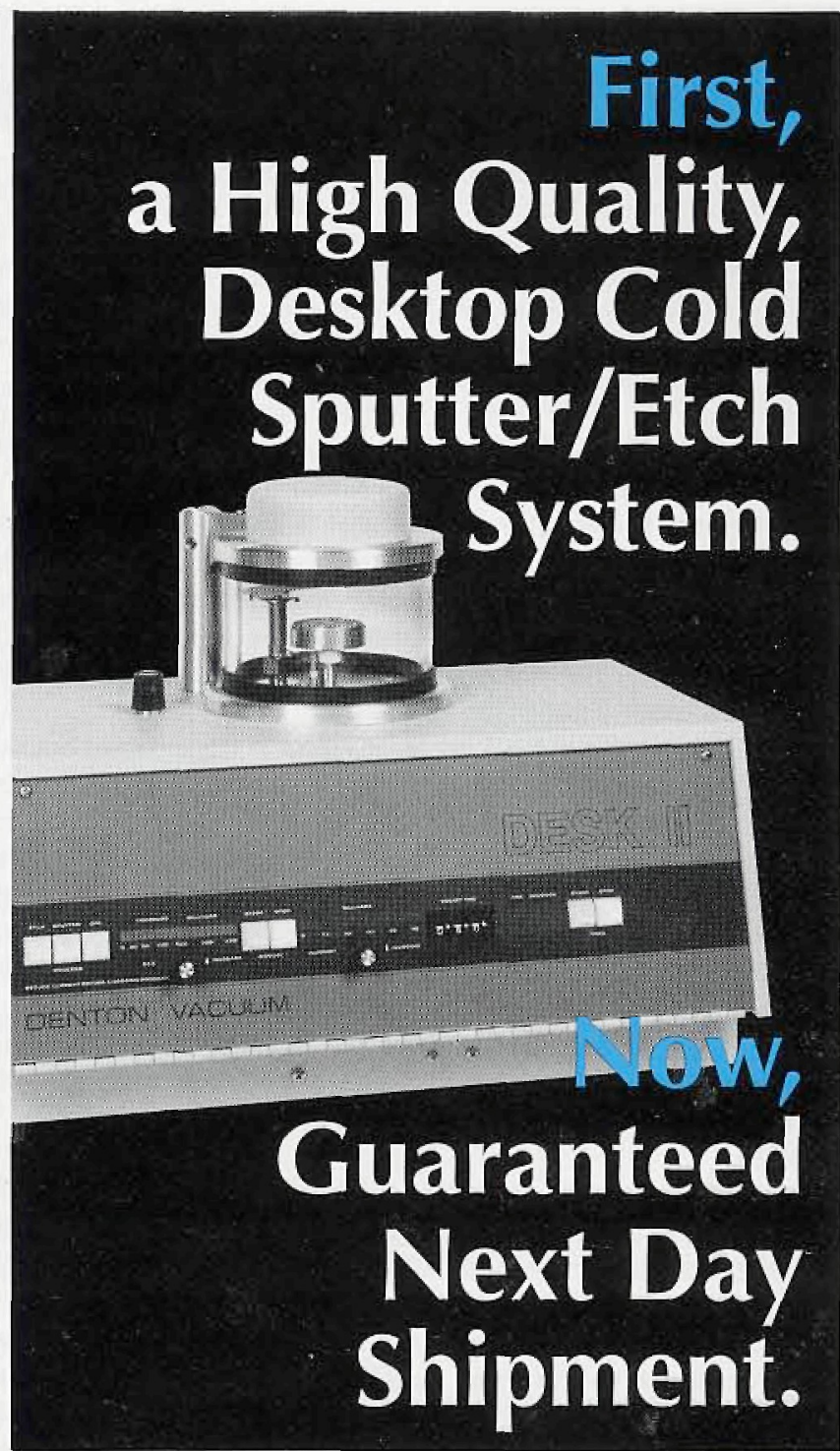

Order Denton's standard Desk II System by noon today and get guaranteed shipment tomorrow or Denton pays the freight.

The Desk II produces uniform, conductive, fine grain $\mathrm{Au} / \mathrm{AuPd}$ coatings in under three minutes and is the highest quality desktop system available.

\section{The Desk // features:}

- Automatic or manual operation

Easy to read digital vacuum and current gauges

- Optional carbon evaporation accessory

For more information, look no further than Denton... where both Quality and Delivery invite comparison.

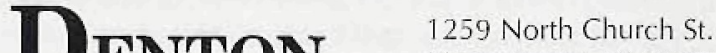

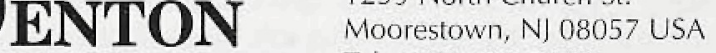 \\ VACUUM I INC.

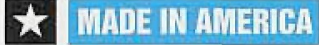

Next day shipment must be requested at time of order.

Decades of Experience In EM Specimen Prep Equipment 